\title{
Práticas Relevantes em Engenharia de Software: uma avaliação de especialistas
}

\author{
Mauro Oddo Nogueira \\ COPPE/UFRJ \\ mauroddo@urbi.com.br
}

\author{
Prof ${ }^{a}$. Ana Regina Cavalcanti da Rocha \\ COPPE/UFRJ \\ darocha@cos.ufrj.br
}

\begin{abstract}
Resumo
O Ministério da Ciência e Tecnologia - MCT do Brasil, através da Secretaria de Política de Informática e Automação - SEPIN, realiza, bienalmente, uma pesquisa que objetiva avaliar o perfil da indústria brasileira de software no que se refere à Qualidade. Essa pesquisa é conduzida através de um questionário distribuído a uma parcela significativa da indústria. Nele, são relacionadas práticas relativas à Engenharia de Software, documentação e ferramentas. Todavia, não há consenso em relação a quais destas são, efetivamente, relevantes para a Qualidade do software. O presente trabalho consiste em uma pesquisa de campo com especialistas que objetiva identificar quais dessas práticas, documentações e ferramentas de fato impactam a Qualidade do software. Seus resultados permitem, além de aprimorar a própria pesquisa SEPIN, balizar a atuação dos gerentes e desenvolvedores, auxiliando-os na conformação de seus processos de software.

Palavras-Chave: Qualidade de Software; Engenharia de Software; Documentação; Ferramentas.
\end{abstract}

\begin{abstract}
The Ministério da Ciência e Tecnologia - MCT do Brasil, through the Secretaria de Política de Informática e Automação - SEPIN, accomplishes, every two years, a research that aims at an evaluation of the Brazilian software industry profile, where it concerns Quality. This research is carried out through a form, which is handed out to a significant portion of the industry. In it, Software Engineering practices, documentation and tools are listed. However, there is no consensus to which of these are effectively relevant to the software quality. This essay, consisting in a survey with specialists, has in view identify which of these practices, documentation and tools can really affect software quality. Its results allow not only the improvement of the SEPIN research, but also aid managers and developers, helping them in the conforming of their software processes.

Key-Words: Software Quality; Software Engineering; Documentation; Tools.
\end{abstract}

\section{Introdução}

O esforço da indústria brasileira de software na busca pela qualidade tem sido objeto de pesquisa realizada a cada dois anos pelo Ministério da Ciência e Tecnologia, através da Secretaria de Política de Informática e Automação - SEPIN. Seus resultados estão divulgados em um documento intitulado "Qualidade e Produtividade no Setor de Software Brasileiro - 2001" [2].

Nessa pesquisa, um conjunto de questões busca identificar as práticas das empresas no que se refere à Engenharia de Software. São elas: as práticas adotadas para desenvolvimento e manutenção; a documentação adotada; as ferramentas e as práticas utilizadas para avaliação da qualidade do produto.

O questionário apresenta variado elenco dessas técnicas e ferramentas, cabendo a cada empresa participante assinalar aquelas que são por ela utilizadas. A indicação da adoção dessas práticas é entendida como indicadores para a verificação do uso das boas práticas de Engenharia de Software nas empresas brasileiras. Todavia, é de se supor que nem todo o elenco de opções possa ser considerado como igualmente relevante. Quais são, portanto, entre as práticas elencadas, as melhores práticas de Engenharia de Software? Existe pouca comprovação empírica sobre a eficiência e eficácia das práticas de Engenharia de Software propostas na literatura e que constituem o estado da arte e da prática [6,7].

Buscando-se identificar quais seriam as técnicas e ferramentas de Engenharia de Software que são, de fato, relevantes para a Qualidade e para a Engenharia de Software - realizou-se uma 
pesquisa de campo junto a especialistas com este objetivo.

O capítulo 2 do presente trabalho descreve a metodologia da pesquisa; o capítulo 3, o perfil da amostra obtida e o processo de consolidação. No capitulo 4 são apresentados os resultados do estudo e, no capítulo 5, as conclusões.

\section{Metodologia da Pesquisa}

A pesquisa foi estruturada segundo o modelo "Goal, Question, Metric" [4] e a metodologia proposta por WOHLIN et al. [5], descritos seguir.

\subsection{Objetivos}

Foi delineado como objetivo global:

"Identificar quais as práticas de Engenharia de Software, ferramentas e documentação relacionadas no questionário da SEPIN devem ser consideradas como capazes de influenciar positivamente a qualidade do processo e/ou do produto de software".

\section{E como objetivo específicos:}

Analisar o conjunto de Práticas de Engenharia de Software adotadas nos processos de desenvolvimento e manutenção de software, oferecido para escolha no questionário da SEPIN, e,

Com o propósito de verificar

Com respeito a sua relevância para a qualidade do processo e/ou produto de software Do ponto de vista dos especialistas em Qualidade de Software e Engenharia de Software No contexto de sua utilização no desenvolvimento de software

Analisar o conjunto de Ferramentas, oferecido para escolha no questionário da SEPIN, e, Com o propósito de verificar

Com respeito a sua relevância para a qualidade do processo e/ou produto de software Do ponto de vista dos especialistas em Qualidade de Software e Engenharia de Software No contexto de sua utilização no desenvolvimento de software

Analisar o conjunto de Documentação, oferecido para escolha no questionário da SEPIN, e, Com o propósito de verificar

Com respeito a sua relevância para a qualidade do processo e/ou produto de software Do ponto de vista dos especialistas em Qualidade de Software e Engenharia de Software No contexto de sua utilização no desenvolvimento de software

Analisar o conjunto de Práticas de Engenharia de Software adotadas na avaliação da qualidade do produto, oferecido para escolha no questionário da SEPIN

Com o propósito de verificar

Com respeito a sua relevância para a qualidade do processo e/ou produto de software Do ponto de vista dos especialistas em Qualidade de Software e Engenharia de Software No contexto de sua utilização no desenvolvimento de software

\subsection{Quadro de Referência}

Objeto de estudo: itens do questionário da SEPIN relativos a:

(i) Práticas de Engenharia de Software adotas pela organização no desenvolvimento e manutenção de software: 
(ii) Ferramentas utilizadas;

(iii) Documentação adotada;

(iv) Práticas de Engenharia de Software adotadas pela organização na avaliação da qualidade do produto.

Perspectiva: A perspectiva é dos especialistas em Qualidade de Software e Engenharia de Software.

Contexto: $\mathrm{O}$ estudo foi conduzido através da coleta de opinião de diversos especialistas atuantes em variados contextos empresariais e acadêmicos, com diversos níveis de formação e experiência profissional. O conjunto de itens foi apresentado através de questionário específico entregue aos participantes.

\subsection{Questões e Métricas}

Foram identificadas quatro questões, onde:

PQ: conjunto de práticas/documentação ou ferramentas do questionário da SEPIN.

PR: conjunto de práticas/ documentação ou ferramentas consideradas como relevantes.

(i) Existem práticas de Engenharia de Software para o desenvolvimento e manutenção de softwares, apresentadas no questionário da SEPIN, cuja relevância para a qualidade do processo e/ou produto de software deva ser desconsiderada?

Hipótese nula $1\left(\mathrm{H}_{0} 1\right)$ : O conjunto de práticas apresentado é, em sua totalidade, relevante; ou seja, nenhuma prática deve ser desconsiderada. - $H_{o} 1: P Q_{1}-P R_{1}=\varnothing$

Hipótese Alternativa 1 (H1): Existem práticas no questionário que não possuem relevância para a qualidade do processo e/ou produto de software. - H1: $P Q_{1}-P R_{1} \neq \varnothing$

Métricas: A lista de práticas consideradas como relevantes pelos especialistas consultados.

(ii) Existem Ferramentas, apresentadas no questionário da SEPIN, cuja relevância para a qualidade do processo e/ou produto de software deva ser desconsiderada?

Hipótese nula $2\left(\mathrm{H}_{0} 2\right)$ : $\mathrm{O}$ conjunto de Ferramentas apresentado é, em sua totalidade, relevante; ou seja, nenhuma ferramenta deve ser desconsiderada. $-H_{o} 2: P Q_{2}-P R_{2}=\varnothing$ Hipótese Alternativa 2 (H2): Existem Ferramentas no questionário que não possuem relevância para a qualidade do processo e/ou produto de software. - $H 2: P Q_{2}-P R_{2} \neq \varnothing$ Métricas: A lista de ferramentas consideradas como relevantes pelos especialistas consultados.

(iii) Existe Documentação, apresentada no questionário da SEPIN, cuja relevância para a qualidade do processo e/ou produto de software deva ser desconsiderada?

Hipótese nula $3\left(\mathrm{H}_{0} 3\right)$ : $\mathrm{O}$ conjunto de Documentação apresentado é, em sua totalidade, relevante; ou seja, nenhuma documentação deve ser desconsiderada. $-H_{o} 3: P Q_{3}-P R_{3}=\varnothing$

Hipótese Alternativa 3 (H3): Existe Documentação no questionário que não possui relevância para a qualidade do processo e/ou produto de software. - $H 3: P Q_{3}-P R_{3} \neq \varnothing$ Métricas: A lista de documentação considerada como relevante pelos especialistas consultados.

(iv) Existem práticas de Engenharia de Software para a avaliação da qualidade do produto, apresentadas no questionário da SEPIN, cuja relevância para a qualidade do processo e/ou produto de software deva ser desconsiderada?

Hipótese nula $4\left(\mathrm{H}_{0} 4\right)$ : O conjunto de práticas apresentado é, em sua totalidade, relevante; ou seja, nenhuma prática deve ser desconsiderada. $-H_{0} 4: P Q_{4}-P R_{4}=\varnothing$ 
Hipótese Alternativa 4 (H4): Existem práticas no questionário que não possuem relevância para a qualidade do processo e/ou produto de software. - $H 4: P Q_{4}-P R_{4} \neq \varnothing$

Métricas: A lista de práticas consideradas como relevantes pelos especialistas consultados.

\subsection{Instrumentação}

Como instrumentação da pesquisa, foi elaborado um questionário que reproduz as opções do questionário da SEPIN. Trata-se de um questionário fechado de opções múltiplas, com uma questão aberta para cada conjunto de respostas. Os resultados das questões abertas não serão considerados para a análise a que se propõe este estudo. Porém serão objetos de uma analise complementar que poderá futuramente contribuir para o aprimoramento do questionário da SEPIN. O questionário inclui ainda quesitos que têm por objetivo caracterizar e qualificar o especialista participante em relação à atividade que desempenha, sua formação e sua experiência profissional, de modo a hierarquizá-los para atribuição de peso às suas respostas.

\subsection{Seleção de Contexto}

Os questionários foram entregues a cada participante para preenchimento sem acompanhamento. Para tanto, foram utilizados questionários em meio físico, que foram entregues pessoalmente e em meio eletrônico, distribuídos através de e-mails, via Internet. O preenchimento foi voluntário e realizado no tempo e ambiente escolhidos pelo participante.

\subsection{Seleção de Indivíduos}

Os indivíduos foram selecionados por conveniência e disponibilidade, não tendo sido aplicado nenhum método estocástico. Todavia, foi envidado o máximo esforço no sentido de abranger-se uma parcela consideravelmente representativa do universo de especialistas em Qualidade e Engenharia de Software no Brasil. Assim sendo, procurou-se obter a colaboração de indivíduos da indústria e da academia, de diversas regiões do país e de diversas experiências.

Deste modo, participaram estudantes de cursos de pós-graduação de universidades de vários Estados e com variados perfis discentes; membros de entidades filiadas à Sociedade Softex; professores de diversas Instituições de Ensino Superior, tanto de Graduação quanto de pósgraduação; profissionais atuantes nos diversos ramos da indústria; participantes do Sub-comitê Setorial de Software do Programa Brasileiro de Qualidade e Produtividade - SSQP/PBQP, que se caracteriza por apresentar uma composição mista, envolvendo universidades, indústrias e governo (o questionário foi distribuído por meio eletrônico aos participantes do Sub-comitê, através de sua "mailing list" e por meio físico, em duas reuniões deste grupo, realizadas em $2002 \mathrm{em}$ Araras, RJ e Gramado, RS); e a diversos participantes do XV Simpósio Brasileiro de Engenharia de Software (XV SBES) e I Simpósio Brasileiro de Qualidade de Software (I SBQS), ambos em Gramado, RS.

\subsection{Variáveis}

Variáveis independentes:

- Conjunto das Práticas de Engenharia de Software adotadas no processo desenvolvimento e manutenção de software;

- Conjunto de Ferramentas;

- Conjunto de Documentação;

- Conjunto de Práticas de Engenharia de Software adotadas na avaliação da qualidade do produto.

Variáveis dependentes:

- Conjunto das Práticas de Engenharia de Software adotadas no processo desenvolvimento e manutenção de software capazes de influenciar positivamente a qualidade em processo e/ou produto de software; 
- Conjunto de Ferramentas capazes de influenciar positivamente a qualidade em processo e/ou produto de software;

- Conjunto de Documentação capazes de influenciar positivamente a qualidade em processo e/ou produto de software;

- Conjunto de Práticas de Engenharia de Software adotadas na avaliação da qualidade do produto capazes de influenciar positivamente a qualidade em processo e/ou produto de software.

\subsection{Validade}

Validade do Constructo:

Os conjuntos de Práticas, Ferramentas e Documentação apresentados no questionário utilizado na pesquisa de campo reproduzem aqueles apresentados como opção no questionário da SEPIN. Além disso, a própria apresentação do questionário também reproduziu aquela utilizada pela SEPIN. Desta forma, espera-se que a percepção do questionário por parte dos participantes da pesquisa seja análoga à percepção dos respondentes das organizações participantes.

\section{Validade Interna:}

No estudo realizado, foram recolhidas 231 contribuições; com um nível de significância de $5 \%$ e considerando-se uma distribuição binomial de população desconhecida, o erro amostral calculado foi da ordem de 6,45\%. A amostra que foi de fato utilizada é composta por 166 especialistas. Esta redução no tamanho da amostra, contudo, antes de reduzir a confiabilidade do resultado do estudo, contribui sobremaneira para aumentá-la, já que assegura sua representatividade. As justificativas desta análise são apresentadas em seção subseqüente, em que se tratará da validade externa.

Para um perfeito entendimento dos aspectos concernentes à validade interna, é importante notar-se que não se trata de uma "pesquisa de opinião" na qual pretende-se inferir o "comportamento" do universo. Ou seja, a amostra não foi construída de forma aleatória, mas sim a partir da seleção dos indivíduos do universo cujas características os habilitam como especialistas na área. Trata-se, portanto, de uma avaliação por especialistas, na qual os participantes foram selecionados em função de sua atuação profissional, qualificação e experiência. Além disso, as respostas fornecidas por cada especialista são ponderadas em função de sua qualificação e experiência na área. Neste caso, a distribuição da amostra em função desses fatores é o que de fato determina a confiabilidade dos resultados, e não exclusivamente o tamanho da amostra. Assim, relevante não é a significância estatística da amostra, mas sim a sua conformação.

Considerando-se os especialistas participantes, assume-se que a amostra construída é representativa do universo estudado, conferindo-lhe a confiabilidade necessária. Vale destacar que a quase totalidade dos mais notórios especialistas do setor no país tomou parte do estudo.

\section{Validade Externa}

Os participantes foram escolhidos em função de sua notoriedade e de seu envolvimento nas comunidades de Qualidade e Engenharia de Software brasileiras. Sua representatividade pode ser avaliada a partir dos dados de caracterização dos indivíduos fornecidos nos questionários e que se encontram descritos em uma tabela específica quando da apresentação dos resultados.

As condições oferecidas para resposta - possibilidade de respondê-lo no tempo e ambiente que cada participante considerar adequado - pode resultar em uma ameaça à validade externa, uma vez que tais aspectos podem influenciar as respostas dadas.

Um outro aspecto que poderia vir a comprometer a validade externa do estudo relaciona-se aos métodos utilizados para a seleção dos participantes e para a distribuição dos questionários. Em 
virtude de restrições decorrentes da própria forma como os especialistas "alvo" foram identificados e contatados, a amostra acabou sendo composta também por elementos cuja qualificação não permite que sejam classificados como "especialistas". A fim de contornar essa questão, a base de dados utilizada foi objeto de um processo de expurgo que assegurou que sua composição refletisse o perfil desejado. As condições que conduziram a esse expurgo, bem como os critérios utilizados serão descritos em seções subseqüentes do presente capítulo.

\section{Validade de Conclusão}

A verificação das hipóteses se dá pela avaliação da indicação de relevância de cada um dos fatores considerados em cada uma das métricas. O conjunto de fatores considerados como relevantes foi construído a partir da frequiência ponderada com que os participantes da pesquisa indicaram a capacidade desses fatores de influenciar na qualidade de produto e/ou processo de software. A ponderação considera a representatividade do participante, tendo em vista sua atuação profissional e/ou acadêmica, qualificação e experiência. Como já foi dito, o conjunto de especialistas participantes apresenta um elevado grau de qualificação e experiência e notoriedade. Assim sendo, é possível a generalização dos resultados.

\subsection{Contribuições Adicionais}

No questionário, em cada um dos conjuntos de opções, foi apresentada como alternativa o item "OUTROS", caracterizado como uma questão aberta na qual o especialista poderia indicar outros elementos que considerasse importantes para o item avaliado. Os dados obtidos a partir dessas alternativas não serão computados para efeito da análise deste estudo. Todavia, a agregação de tais informações poderá ser de grande valia para o aprimoramento no futuro do questionário da SEPIN, permitindo a inclusão daqueles fatores cuja indicação foi freqüente. Do mesmo modo, aqueles fatores que constam das opções oferecidas pelo questionário e cuja frequiência de escolha se mostrar excessivamente baixa, poderão ter sua permanência no questionário avaliada quando de sua revisão.

\section{Consolidação da Pesquisa}

A seguir, é apresentada a metodologia e critérios utilizados para a consolidação da pesquisa, bem como uma descrição do perfil da amostra obtida.

\subsection{Perfil da Amostra Inicial}

No questionário distribuído, para fim de caracterização de cada especialista participante - e determinação da representatividade relativa (Peso) de suas respostas - foram incluídas questões que identificam que tipos de atividades desenvolvem na indústria de software e/ou na universidade; formação acadêmica e respectiva área de formação; tempo de experiência na área de desenvolvimento de software e o número de projetos nos quais tomaram parte. Os resultados agregados destas questões permitem caracterizar o perfil da amostra obtida. As tabelas 1 a 4 mostram, no que se refere à amostra inicial, respectivamente: Participantes por Área de Atuação, Participantes por Atuação em Empresas, Participantes por Atuação em Universidades e Participantes por Formação Acadêmica. Nas tabelas 2 e 3, o total dos valores apresentados ultrapassa o total de participantes do estudo (231), uma vez que o questionário admite múltiplas respostas; ou seja, diversos participantes exercem mais de uma atividade distinta no mesmo segmento. Na tabela 4 foi considerado nível de formação mais alto de cada um dos participantes, uma vez que o questionário permitia múltiplas respostas. 
Tahela 1 - Particinantes nor Área de Atuacão (Amostra Inicial)

\begin{tabular}{|l|l|}
\hline Total de participantes: & $\mathbf{2 3 1}$ \\
\hline Participantes com atuação na indústria: & 167 \\
\hline Participantes com atuação na universidade: & 152 \\
\hline Participantes com atuação exclusiva na indústria: & 64 \\
\hline Participantes com atuação exclusiva na universidade: & 79 \\
\hline Participantes com atuação concomitante em ambas: & 88 \\
\hline
\end{tabular}

Tabela 2 - Participantes por Atuação em Empresas (Amostra Inicial)

\begin{tabular}{|l|l|}
\hline \multicolumn{2}{|c|}{ Empresa } \\
\hline Empresário & 23 \\
\hline Gerente de Informática & 17 \\
\hline Gerente da Qualidade & 16 \\
\hline Gerente de Projeto & 48 \\
\hline Analista de Sistemas & 77 \\
\hline Consultor & 13 \\
\hline
\end{tabular}

Tabela 3 - Participantes por Atuação em Universidades (Amostra Inicial)

\begin{tabular}{|l|c|}
\hline \multicolumn{2}{|c|}{ Universidade } \\
\hline Professor & 74 \\
\hline Pesquisador & 31 \\
\hline Consultor & 17 \\
\hline Aluno de Doutorado & 23 \\
\hline Aluno de Mestrado & 53 \\
\hline Aluno de Graduação & 17 \\
\hline
\end{tabular}

Tabela 4 - Participantes por Formacão Acadêmica (Amostra Inicial)

\begin{tabular}{|l|c|l|c|}
\hline \multicolumn{4}{|c|}{ FORMAÇÃO ACADÊMICA } \\
\hline \multicolumn{2}{|c|}{ Nível } & \multicolumn{2}{c|}{ Área } \\
\hline \hline Doutorado & 40 & Engenharia de Software & 63 \\
\hline Mestrado & 49 & Computação / Informática & 142 \\
\hline Especialização & 40 & Outro & 26 \\
\hline Graduação & 82 & & \\
\hline Certificação em Qualidade & 3 & & 231 \\
\hline Nível Médio & 17 & & \\
\hline \hline Total & 231 & Total & \\
\hline
\end{tabular}

No que se refere à experiência profissional, o tempo médio de atuação em desenvolvimento de software foi 11,4 anos e a mediana do número de projetos em que tomou parte, 10,0 projetos.

A observação dos quadros evidencia a heterogeneidade da amostra no que tange a área de atuação (Indústria/Universidade) e as atividades desempenhadas. Pode-se notar, inclusive, que um contingente significativo destes ( 88 pessoas $\approx 38 \%$ da amostra) desempenha atividades em ambos os contextos. Esta situação contribui para a redução de um possível viés na amostra decorrente das visões particulares de cada segmento. Aliando-se a consideração das atividades exercidas com as qualificações acadêmicas, percebe-se que a amostra é composta, em sua maioria, por profissionais com a representatividade desejada. Não obstante, observa-se que, em decorrência do método de coleta, diversos participantes não podem ser considerados como especialistas (ex.: 17 Alunos de Graduação / Nível Médio; 82 apenas com Graduação; 26 com formação em Outras Áreas). O questionário foi publicamente distribuído em alguns eventos que, por sua natureza, apresentam elevado potencial como concentradores de especialistas, porém, tais eventos também agregam pessoas - particularmente estudantes - que, a despeito de se interessarem pela área, não se caracterizam como especialistas. Como a distribuição foi pública, não seria razoável impedir a colaboração voluntária dessas pessoas. Contudo, conforme será apresentado em uma seção posterior deste trabalho, os mesmos foram "expurgados" da amostra, de modo a elevar sua representatividade. 


\subsection{Cálculo do Peso Individual}

Uma vez que a amostra é heterogênea em relação aos fatores considerados determinantes para a caracterização dos especialistas, deve-se considerar que a relevância de suas respostas também o é. Assim sendo, os participantes foram diferenciados em função desses fatores, tendo sido atribuído um peso individual para cada um deles, calculado em função desses fatores. A fórmula utilizada para a definição dos pesos é:

onde:

$$
P(i)=\operatorname{Máx}\{\operatorname{Máx}[I(i)] ; \operatorname{Máx}[U(i)]\}+F_{1}(i)+F_{2}(i)+T(i)+N(i)
$$

- $\quad P(i)$ é o peso atribuído ao participante $i$;

- $I(i)$ é a pontuação atribuída ao participante $i$ em função de sua atuação na indústria de desenvolvimento de software segundo a tabela 5;

- $U(i)$ é a pontuação atribuída ao participante $i$ em função de sua atuação na universidade segundo a tabela 6

- $F_{1}(i)$ é a pontuação atribuída ao participante $i$ em função de seu nível de formação acadêmica segundo a tabela 7 ;

- $\quad F_{2}(i)$ é a pontuação atribuída ao participante $i$ em função da área de concentração de sua formação acadêmica segundo a tabela 8;

- $T(i)$ é a pontuação atribuída ao participante $i$ em função de seu tempo de atuação na área segundo a tabela 9;

- $N(i)$ é a pontuação atribuída ao participante $i$ em função do número de projetos nos quais tomou parte segundo a tabela 10 .

Tabela 5 - Pontuacão Indústria

\begin{tabular}{|l|c|}
\hline Atuação na Indústria & $\boldsymbol{I}(\boldsymbol{i})$ \\
\hline Empresário & 4 \\
\hline Gerente de Informática & 4 \\
\hline Gerente da Qualidade & 3 \\
\hline Gerente de Projeto & 2 \\
\hline Analista de Sistemas & 1 \\
\hline Consultor & 4 \\
\hline
\end{tabular}

Tabela 7 - Pontuação Nível de Formação

\begin{tabular}{|l|c|}
\hline \multicolumn{1}{|c|}{ Nível de Formação } & $\boldsymbol{F}_{\boldsymbol{I}}(\boldsymbol{i})$ \\
\hline Doutorado & 4 \\
\hline Mestrado & 3 \\
\hline Especialização & 2 \\
\hline Graduação & 1 \\
\hline Certificação & 3 \\
\hline Nível Médio & 0 \\
\hline
\end{tabular}

Tabela 9 - Pontuação Número de Projetos

\begin{tabular}{|l|c|}
\hline Número de Projetos & $\boldsymbol{N}(\boldsymbol{i})$ \\
\hline A partir de 10 & 4 \\
\hline De 4 a 9 & 2 \\
\hline Até 3 & 0 \\
\hline
\end{tabular}

Tabela 6 - Pontuação Universidade

\begin{tabular}{|l|c|}
\hline Atuação na Universidade & $\boldsymbol{U}(\boldsymbol{i})$ \\
\hline Professor & 4 \\
\hline Pesquisador & 3 \\
\hline Consultor & 3 \\
\hline Aluno de Doutorado & 2 \\
\hline Aluno de Mestrado & 1 \\
\hline Aluno de Graduação & 0 \\
\hline
\end{tabular}

Tabela 8 - Pontuação Área de Formação

\begin{tabular}{|l|c|}
\hline \multicolumn{1}{|c|}{ Área de Formação } & $\boldsymbol{F}_{2}(\boldsymbol{i})$ \\
\hline Engenharia de Software & 6 \\
\hline Informática / Computação & 3 \\
\hline Outras áreas & 0 \\
\hline
\end{tabular}

Tabela 10 - Pontuação Tempo de Autuação

\begin{tabular}{|l|c|}
\hline \multicolumn{1}{|c|}{ Tempo de Atuação } & $\boldsymbol{T}(\boldsymbol{i})$ \\
\hline Mais de 15 anos & 6 \\
\hline Acima de 5, até 15 anos & 3 \\
\hline Até 5 anos & 0 \\
\hline
\end{tabular}

Para todas as variáveis que compõem o peso, optou-se pela adoção de tabelas de classes. Para o grupo composto pelas variáveis $I(i), U(i), F_{l}(i)$ e $F_{2}(i)$ isso foi feito por tratarem-se de 
variáveis qualitativas. Deste modo, a cada uma delas foi atribuído um dado valor de modo a permitir seu tratamento quantitativo. Para as demais - T(i) e $N(i)$ - esta opção decorreu do fato de tratar-se de eventos cuja precisa quantificação por parte dos participantes se mostrou difícil; particularmente no caso do Número de Projetos. Além disso, do ponto de vista da caracterização da "importância" do especialista, não se pode atribuir diferenças significativas entre dois profissionais que possuam, por exemplo, 30 e 40 anos de atuação, respectivamente. A adoção do agrupamento por classes permitiu contornar ambos os problemas.

\subsection{Distribuição dos Pesos da Amostra Inicial}

Os pesos calculados para os participantes da amostra inicial apresentaram a Distribuição de Frequiência mostrada no gráfico 1. Dividindo a amostra em quartis, obtêm-se os limites mostrados na tabela 11.

\section{Gráfico 1 - Distribuição de Freqüência dos Pesos (Amostra Inicial)}

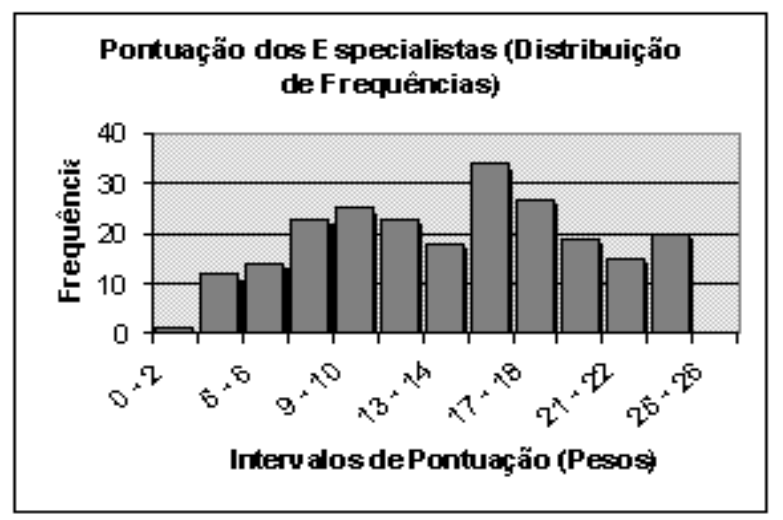

Tabela 11 - Quartis (Amostra Inicial)

\begin{tabular}{|c|c|}
\hline Quartis & Pesos \\
\hline Valor mínimo & 2,0 \\
\hline Limite do $1^{0}$ Quartil & 9,0 \\
\hline Limite do $2^{\circ}$ Quartil & 14,0 \\
\hline Limite do $3^{0}$ Quartil & 18,0 \\
\hline Limite do $4^{\circ}$ Quartil (Max.) & 24,0 \\
\hline
\end{tabular}

\subsection{Ajuste da Amostra}

Conforme discutido anteriormente, em virtude do método de coleta de questionários adotado, vários daqueles que contribuíram com o estudo não são "especialistas". A metodologia acima descrita para a diferenciação dos participantes permite identificá-los, uma vez que sua pontuação será menor do que a dos demais. Este grupo é considerado como representado pelo conjunto de participantes que compõem o $1^{0}$ Quartil da amostra. Assim sendo, a fim de assegurar a representatividade da amostra, todos os questionários preenchidos por participantes cuja pontuação (peso) correspondeu a valores iguais ou inferiores ao limite superior do $1^{\underline{0}}$ quartil $(P(i) \leq 9,0)$ foram excluídos da análise. Tal fato resultou na definição de uma nova amostra para o estudo, que será denominada, na continuidade deste trabalho, como Amostra Ajustada.

\subsection{Perfil da Amostra Ajustada}

Uma vez excluídos os participantes que se enquadram no critério acima definido, tem-se um novo perfil para a amostra utilizada na análise e na determinação dos resultados do estudo. As tabelas 12 a 15 mostram, no que se refere à amostra ajustada, respectivamente: Participantes por Área de Atuação, Participantes por Atuação em Empresas, Participantes por Atuação em Universidades e Participantes por Formação Acadêmica. A despeito do intervalo quartílico da amostra inicial ser igual a 57,75, o número total de participantes com pesos iguais ou menores que 9,0 foi de 65; sendo este o total de participantes excluídos do estudo. Assim, a amostra ajustada representa apenas $71.8 \%$ da amostra inicial. 
Tabela 12 - Participantes por Área de Atuação (Amostra Ajustada)

\begin{tabular}{|l|c|}
\hline Total de participantes: & $\mathbf{1 6 6}$ \\
\hline Participantes com atuação na indústria: & 125 \\
\hline Participantes com atuação na universidade: & 103 \\
\hline Participantes com atuação exclusiva na indústria: & 41 \\
\hline Participantes com atuação exclusiva na universidade: & 63 \\
\hline Participantes com atuação concomitante em ambas: & 62 \\
\hline
\end{tabular}

Tabela 13 - Participantes por Atuação em Empresas (Amostra Ajustada)

\begin{tabular}{|l|c|}
\hline \multicolumn{2}{|c|}{ Empresa } \\
\hline Empresário & 22 \\
\hline Gerente de Informática & 15 \\
\hline Gerente da Qualidade & 11 \\
\hline Gerente de Projeto & 43 \\
\hline Analista de Sistemas & 46 \\
\hline Consultor & 12 \\
\hline
\end{tabular}

Tabela 14 - Participantes por Atuação na Universidade (Amostra Ajustada)

\begin{tabular}{|l|c|}
\hline \multicolumn{2}{|c|}{ Universidade } \\
\hline Professor & 73 \\
\hline Pesquisador & 30 \\
\hline Consultor & 17 \\
\hline Aluno de Doutorado & 21 \\
\hline Aluno de Mestrado & 22 \\
\hline Aluno de Graduação & 2 \\
\hline
\end{tabular}

Tahela 15 - Particinantes nor Formacão Acadêmica (Amostra A iustada)

\begin{tabular}{|l|c|c|l|l|c|c|}
\hline \multicolumn{7}{|c|}{ NORMAÇÃO ACADÊMICA } \\
\hline \multicolumn{2}{|c|}{ Nível } & 40 & $24,1 \%$ & Engenharia de Software & 62 & $37,4 \%$ \\
\hline \hline Doutorado & 45 & $27,1 \%$ & Computação / Informática & 85 & $51,2 \%$ \\
\hline Mestrado & 33 & $19,9 \%$ & Outro & 19 & 11,4 \\
\hline Especialização & 43 & $25,9 \%$ & & \multicolumn{3}{c|}{} \\
\hline Graduação & 3 & $1,8 \%$ & & 166 & $100 \%$ \\
\hline Certificação em Qualidade & 2 & $1,2 \%$ & & Total \\
\hline Nível Médio & 166 & $100 \%$ & & \\
\hline Total & & & & \\
\hline
\end{tabular}

No que se refere à experiência profissional, o tempo médio de atuação em desenvolvimento de software foi 14,4 anos e a mediana do número de projetos em que tomou parte, 12,0 projetos.

Os pesos calculados para os participantes da amostra ajustada apresentaram a Distribuição de Frequiência mostrada no gráfico 2.

Gráfico 2 - Distribuição de Freqüência dos Pesos (Amostra Ajustada)

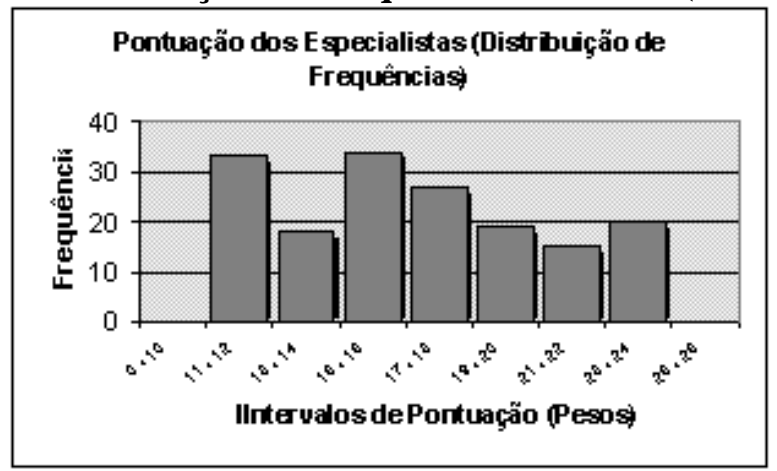

Essa nova amostra mantém as qualidades relacionadas à heterogeneidade de sua composição identificadas na amostra inicial, contando com um índice de participantes que atuam concomitantemente na indústria e na universidade muito próximo ao daquela $(\approx 37 \%)$. Portanto, foram mantidas as características que contribuem para a redução de um possível viés na amostra decorrente das visões particulares de cada segmento. A análise da composição da amostra em 
relação à atuação dos participantes demonstra sua efetiva representatividade. A grande maioria ocupa, na indústria, cargos de gerência e, na universidade, a função de professor. O mesmo se depreende da observação da formação acadêmica dos participantes considerados para esta amostra. Apenas $27 \%$ destes não possuem nenhum tipo de pós-graduação e destes, somente 2 (1,2\% da amostra) não possuem curso de Graduação; estão ambos cursando-a atualmente, porém possuem larga experiência (tanto em termos de tempo, quanto de variedade) na área e ocupam posições de gerentes nas empresas em que trabalham. A nova amostra passou a apresentar um tempo médio de atuação na área de 14,4 anos e a mediana do número de projetos subiu para 12 . Valores que, aliados à formação acadêmica e ao nível de atuação profissional, se apresentam como bastante significativos. Merece ainda destaque o fato de que praticamente a totalidade dos profissionais, professores e pesquisadores de maior destaque na comunidade envolvida com Qualidade de Software no Brasil tomou parte na pesquisa. Este conjunto de características imprime à amostra considerada no estudo o nível de representatividade que permite assumir-se a validade de seus resultados.

\subsection{Cálculo dos Pesos Agrupados}

Quando do cálculo do peso individual de cada um dos especialistas, os valores atribuídos a cada um dos fatores considerados foram determinados a partir de números escalares associados a classes de eventos. Todavia, a comparação entre os pesos individuais obtidos por tal tratamento pode levar a algumas distorções, particularmente quando o enquadramento de um dado participante em uma das classes ocorreu a partir dos valores limites dessas classes. Como exemplo, podem ser citados dois participantes hipotéticos, um com 15 anos de experiência e outro com 16. Neste caso, a despeito de na realidade não existir uma diferença significativa entre suas experiências profissionais, o segundo recebeu o dobro da pontuação do primeiro nesse quesito (3 e 6 pontos, respectivamente). Acresça-se a isso o fato de que a resposta para estas perguntas por parte de muitos dos participantes (especialmente àquela que se refere ao Número de Projetos) foi feita com algum grau de incerteza; o que também pode levar, especialmente nos limites das classes, a transposição desta incerteza para o valor a ele atribuído. Mesmo nas variáveis qualitativas, situações semelhantes podem ocorrer. Veja-se, como exemplo, dois estudantes de doutorado. Suponha-se que o primeiro deles tenha recém ingressado no curso, ao passo que o segundo já tenha terminado sua tese, estando aguardando apenas a sua defesa. Pelo tratamento dado, ambos receberiam a mesma pontuação (1 ponto). É evidente que há uma notável diferença na representatividade das respostas dadas por cada um deles e que não ficou representada nas respectivas pontuações.

Este problema foi solucionado pelo agrupamento dos especialistas em classes, de modo a adotar-se um peso único para todos aqueles que pertencem a uma dada classe. Assim, assumindose um peso representativo da classe, elimina-se as possíveis distorções que resultariam da comparação individual entre os pesos de cada especialista, considerando-se como "não significativas" as diferenças dos pesos individuais entre os especialistas de uma mesma classe. Como critério para o estabelecimento destas classes, novamente optou-se pela divisão da amostra em seus Quartis (tabela 16), onde cada limite superior dos Quartis foi utilizado como delimitador das respectivas classes.

É importante chamar a atenção para o fato de que, a despeito do intervalo quartílico para uma amostra de 166 elementos ser igual a 41,5, este não foi o tamanho exato de cada uma das classes consideradas. Isso se deveu ao fato dos pesos se tratarem de valores escalares de tal modo que diversos "eventos" possuem o valor correspondente ao limite dos quartis, pertencendo, portanto,

alguns deles a este quartil e os demais ao quartil subseqüente. Quando do estabelecimento das classes, todos os "eventos" com o mesmo valor são classificados dentro de uma mesma classe, o que gera essa diferença. 
Tabela 16 - Quartis (Amostra Ajustada)

\begin{tabular}{|c|c|}
\hline Quartis & Pesos \\
\hline Valor mínimo & 10,0 \\
\hline Limite do $1^{0}$ Quartil & 14,0 \\
\hline Limite do $2^{\underline{0}}$ Quartil & 16,0 \\
\hline Limite do $3^{-}$Quartil & 20,0 \\
\hline Limite do $4^{\circ}$ Quartil (Máximo) & 24,0 \\
\hline
\end{tabular}

Na tabela 17 são apresentadas as classes definidas e seus respectivos valores limite, frequiência (Qtd.), valor médio e desvio padrão. Considerando-se a Média e o Desvio Padrão de cada classe, pode-se constatar que cada uma delas apresenta um razoável grau de homogeneidade, o que permite considerar como "não significativas" as diferenças de pesos existentes entre cada um dos seus componentes.

Para o cálculo dos pesos a serem atribuídos a cada uma das classes, utilizou-se a classe "A" como referência, atribuindo-se a ela um peso igual a 1,0. Para as demais, classes, seu peso foi calculado relativamente ao peso da classe "A", de acordo com a fórmula:

$$
P(j)=\frac{\bar{X}(j)}{\bar{X}(A)}
$$

onde:

$P(j)$ é o peso atribuído à classe $j$;

$X(j)$ é o Peso Médio da classe $j$;

$X(A)$ é o Peso Médio da classe $A$.

Assim, obtêm-se os pesos de cada classe conforme a tabela 18.

Tabela 17 - Classes de Especialistas

\begin{tabular}{|c|c|c|c|c|}
\hline Classe & Peso Limite & Qtd. & Peso Médio & $\begin{array}{c}\text { Desvio } \\
\text { Padrão }\end{array}$ \\
\hline A & Até 14,0 & 51 & 12,12 & 1,41 \\
\hline B & De 15,0 a 16,0 & 34 & 15,41 & 0,49 \\
\hline C & De 17,0 a 20,0 & 46 & 18,46 & 1,14 \\
\hline D & Maior que 20,0 & 35 & 22,54 & 1,38 \\
\hline
\end{tabular}

Tabela 18 - Peso por Classe

\begin{tabular}{|c|c|c|}
\hline Classe & Cálculo do Peso & Peso \\
\hline A & 1,0 & 1,00 \\
\hline B & $15,41 / 12,12$ & 1,27 \\
\hline C & $18,46 / 12,12$ & 1,52 \\
\hline D & $22,54 / 12,12$ & 1,86 \\
\hline
\end{tabular}

\section{Resultados}

\subsection{Consolidação dos Resultados}

Para a consolidação dos resultados, cada voto de cada especialista foi computado com base no peso da classe a que pertence. Finalmente, o total dos votos de cada uma das questões foi dividido pelo somatório total dos pesos - valor que foi igual a 229,4 - obtendo-se, assim, a votação proporcional de cada uma das questões. As tabelas 19 a 22 apresentam os resultados da consolidação, com a votação proporcional de cada questão: 
Tabela 19 - Consolidação dos Resultados: Práticas de Engenharia de Software. no Processo Práticas de Engenharia de Software adotadas nos processos de desenvolvimento e manutenção de softwares

\begin{tabular}{|l|c|l|c|}
\hline Análise crítica conjunta & 0,46 & Gerência de risco & $\mathbf{0 , 5 6}$ \\
\hline Controles de versão de produto & $\mathbf{0 , 7 7}$ & Gestão de mudança & 0,44 \\
\hline Engenharia da informação & 0,33 & Joint Application Design - JAD & 0,33 \\
\hline Especificação de programas & $\mathbf{0 , 5 7}$ & Métodos estruturados & 0,46 \\
\hline Especificação de projetos & $\mathbf{0 , 6 7}$ & Métodos orientados a objetos & $\mathbf{0 , 7 1}$ \\
\hline Especificação de requisitos & $\mathbf{0 , 9 1}$ & Modelagem de dados & $\mathbf{0 , 8 1}$ \\
\hline Estimativa de custos & $\mathbf{0 , 6 0}$ & Normas e padrões da organização & $\mathbf{0 , 6 6}$ \\
\hline Estimativa de esforço & $\mathbf{0 , 6 0}$ & Planejamento formal de testes & $\mathbf{0 , 6 9}$ \\
\hline Estimativa de tamanho & 0,43 & Projeto da interface com o usuário & $\mathbf{0 , 7 2}$ \\
\hline Gerência de configuração & $\mathbf{0 , 5 8}$ & Prototipação & $\mathbf{0 , 6 6}$ \\
\hline Gerência de requisitos & $\mathbf{0 , 7 1}$ & & \\
\hline
\end{tabular}

Tabela 20 - Consolidação dos Resultados: Documentação

\begin{tabular}{|l|c|l|c|}
\hline Documentação & $\mathbf{0 , 5 8}$ & Histórico do projeto & $\mathbf{0 , 5 1}$ \\
\hline Acompanhamento de custos & $\mathbf{0 , 7 3}$ & Identificação de risco & 0,47 \\
\hline Acompanhamento de prazos & $\mathbf{0 , 5 5}$ & Manual de treinamento & $\mathbf{0 , 5 3}$ \\
\hline Contratos e acordos & 0,32 & Manual do sistema & $\mathbf{0 , 6 9}$ \\
\hline Descrição do produto para comercialização & 0,16 & Manual do usuário & $\mathbf{0 , 7 2}$ \\
\hline Documentação de marketing & $\mathbf{0 , 7 7}$ & Plano de contingência & $\mathbf{0 , 5 5}$ \\
\hline Documentação de programas & $\mathbf{0 , 7 8}$ & Plano de controle da qualidade & $\mathbf{0 , 6 1}$ \\
\hline Documentação do processo de software & $\mathbf{0 , 7 9}$ & Plano de testes & $\mathbf{0 , 7 6}$ \\
\hline Documentação no código & $\mathbf{0 , 8 6}$ & Projeto do sistema & $\mathbf{0 , 7 0}$ \\
\hline Especificação do sistema & $\mathbf{0 , 5 8}$ & Registro formal de revisões e testes & $\mathbf{0 , 6 2}$ \\
\hline Guia de instalação & $\mathbf{0 , 7 1}$ & Relatório de teste & $\mathbf{0 , 5 6}$ \\
\hline Help on-line &
\end{tabular}

Tabela 21 - Consolidação dos Resultados: Ferramentas

Ferramentas

\begin{tabular}{|l|c|l|c|}
\hline Analisador de cobertura de código & 0,14 & Gerador de relatórios & 0,49 \\
\hline Analisador de código & 0,31 & Gerador de telas & 0,33 \\
\hline CASE Lower & 0,45 & Gerenciador de bibliotecas de módulos & 0,47 \\
\hline CASE Upper & 0,49 & Gerenciador de configuração & $\mathbf{0 , 5 9}$ \\
\hline Depurador interativo & 0,43 & Gerenciador de conteúdo & 0,21 \\
\hline Distribuição de software & 0,23 & Gerenciador de documentos & $\mathbf{0 , 5 1}$ \\
\hline Documentador & $\mathbf{0 , 5 6}$ & Gerenciador de projetos & $\mathbf{0 , 6 6}$ \\
\hline Driver de teste & 0,28 & Otimizador & 0,17 \\
\hline Gerador de código-fonte & 0,38 & Prototipador & 0,30 \\
\hline Gerador de dados de teste & 0,38 & Record \& Playback para testes & 0,19 \\
\hline Gerador de entrada de dados & 0,22 & Teste de performance & 0,45 \\
\hline Gerador de gráficos & 0,20 & Visualização cód./classes ou módulo & 0,38 \\
\hline Gerador de GUI & 0,45 & & \\
\hline
\end{tabular}

Tabela 22 - Consolidação dos Resultados: Práticas de Engenharia de Software. em Qualidade de Produtos

Práticas de Engenharia de Software adotadas na avaliação da qualidade do produto:

\begin{tabular}{|l|c|l|c|}
\hline Auditorias & $\mathbf{0 , 5 0}$ & Testes de aceitação & $\mathbf{0 , 7 8}$ \\
\hline $\begin{array}{l}\text { Inspeção formal, Revisão por pares (Peer- } \\
\text { review), Walthrough estruturado }\end{array}$ & $\mathbf{0 , 6 3}$ & Testes de campo & $\mathbf{0 , 5 7}$ \\
\hline Julgamento de especialistas & 0,34 & Testes de integração & $\mathbf{0 , 7 6}$ \\
\hline Levantamento de requisitos de qualidade & $\mathbf{0 , 6 7}$ & Testes de unidade & $\mathbf{0 , 6 1}$ \\
\hline Medições da qualidade (Métricas) & $\mathbf{0 , 6 8}$ & Testes do sistema integrado & $\mathbf{0 , 7 1}$ \\
\hline Modelos de confiabilidade de software & 0,26 & Testes estruturais & 0,48 \\
\hline Prova formal de programas & 0,17 & Testes funcionais & $\mathbf{0 , 7 5}$ \\
\hline Segurança do produto final & 0,28 & Testes orientados a objetos & 0,41 \\
\hline Testes baseados em erros & 0,45 & Testes para web & 0,45 \\
\hline
\end{tabular}




\subsection{Resultados Finais}

Como critério de corte, foram assumidos como considerados influenciadores relevantes na qualidade do produto e/ou do processo de software por parte dos especialistas, todos os quesitos cuja votação proporcional foi igual ou superior a 0,5 . A saber:

\section{(i) Práticas de engenharia de software adotadas nos processos de desenvolvimento e manutenção de softwares}

- Controles de versão de produto

- Especificação de programas

- Especificação de projetos

- Especificação de requisitos

- Estimativa de custos

- Estimativa de esforço

- Gerência de configuração

- Gerência de requisitos
- Gerência de risco

- Métodos orientados a objetos

- Modelagem de dados

- Normas e padrões da organização

- Planejamento formal de testes

- Projeto da interface com o usuário

- Prototipação

Portanto, a Hipótese nula $1\left(\mathrm{H}_{0} 1\right)$ foi considerada falsa, uma vez que diversas das Práticas de Engenharia de Software para o Desenvolvimento e Manutenção de Software relacionadas na pesquisa da SEPIN foram desconsideradas pelos especialistas.

Documentação

- Acompanhamento de custos

- Acompanhamento de prazos

- Contratos e acordos

- Documentação de programas

- Documentação do processo de software

- Documentação no código

- Especificação do sistema

- Guia de instalação

- Help on-line
- Histórico do Projeto

- Manual de treinamento

- Manual do sistema

- Manual do usuário

- Plano de contingência

- Plano de controle da qualidade

- Plano de testes

- Projeto do sistema

- Registro formal de revisões e testes

- Relatório de teste

Portanto, a Hipótese nula $3\left(\mathrm{H}_{0} 3\right)$ foi considerada falsa, uma vez que diversos elementos de Documentação relacionados na pesquisa da SEPIN foram desconsiderados pelos especialistas.

(iii) Ferramentas

- Documentador

- Gerenciador de configuração
- Gerenciador de documentos

- Gerenciador de projetos

Portanto, a Hipótese nula $2\left(\mathrm{H}_{0} 2\right)$ foi considerada falsa, uma vez que diversas Ferramentas relacionadas na pesquisa da SEPIN foram desconsideradas pelos especialistas.
(iv) Práticas de Engenharia de Software adotadas na avaliação da qualidade do produto
- Auditorias
- Inspeção formal, Revisão por pares
- Testes de aceitação
(Peer-review), Walkthrough estruturado
- Testes de campo
- Testes de integração
- Levantamento de requisitos de qualidade
- Testes de unidade
- Medições da qualidade (Métricas)
- Testes do sistema integrado
- Testes funcionais

Portanto, a Hipótese nula $4\left(\mathrm{H}_{0} 4\right)$ foi considerada falsa, uma vez que diversas Práticas de Engenharia de Software para Avaliação da Qualidade do Produto relacionadas na pesquisa da SEPIN foram desconsideradas pelos especialistas. 


\subsection{Contribuições Adicionais}

Conforme relatado anteriormente, cada uma das perguntas apresentava, no questionário, uma opção que se caracterizava como uma "questão aberta", na qual os participantes poderiam sugerir outros quesitos que não fizessem parte da relação apresentada. Foi feita uma análise qualitativa das respostas, identificando aquelas com um mesmo significado, objetivando consolidá-los sob uma única descrição. Os mais relevantes foram:

- Dentre as Práticas para Desenvolvimento e Manutenção de Software, a única a destacar foi o "Gerenciamento de Projeto". As demais sugestões apresentaram apenas 1 ocorrência.

- Para a Documentação, foram citadas mais de uma vez a "Documentação de Requisitos", os "Registros de Reuniões de Acompanhamento" e o "Plano do Projeto".

- Quanto às Ferramentas, também uma merece destaque, o "Gerenciador de Requisitos".

- Nas Práticas para Avaliação da Qualidade do Produto, foram citados mais de uma vez os "Testes de Usabilidade" e "Testes de Carga".

Como contribuição adicional do presente trabalho, fica a sugestão de que essas contribuições sejam consideradas na revisão do questionário da SEPIN a fim de complementá-lo. É admissível considerar-se, ainda, que em um eventual estudo posterior com especialistas, com o mesmo objetivo deste, este conjunto adicional de quesitos possa vir a ser considerado para fazer parte das opções a serem oferecidas como respostas.

\section{Conclusão}

O presente trabalho indicou um conjunto de práticas de Engenharia de Software, Documentação e Ferramentas consideradas, por especialistas, como influenciadoras na qualidade do produto ou do processo de software. Essa indicação contribui para elucidar controvérsias em relação àquilo que é efetivamente relevante dentro do amplo universo de propostas, sugestões e recomendações nesse campo. Os resultados obtidos oferecem um balizamento para a atuação dos gerentes e desenvolvedores, auxiliando-os para uma conformação de seus processos de software, de modo a aumentar sua eficácia no que tange a qualidade de software.

\section{Referências}

[1] FARIA, Luciana de Landa. Planejamento de Riscos em Ambientes de Desenvolvimento de Software Orientados à Organização. Dissertação de mestrado, COPPE/UFRJ, Rio de Janeiro, 2002.

[2] MCT/SEPIN - Secretaria de Política de Informática e Automação / Ministério da Ciência e Tecnologia. Qualidade e Produtividade no Setor de Software Brasileiro - 2001 / N.4. Brasília, 2002.

[3] LARSON, H J. Introduction to Probability Theory and Statistical Inference. John Wiley \& Sons, Inc., $3^{\text {rd }}$ ed., New York, 1982.

[4] Solingen, R e Berghout, E. The Goal / Question / Metric Method: a practical guide for quality improvement of software development. McGraw-Hill, London, 1999.

[5] WoHlin, C et al. Experimentation in Software Engineering: an introduction. Kluwer Academic Publishers, Norwell, 2000.

[6] Zelkowitz, Marvin V. e Wallace, Dolores R. "Experimental Models for Validating Technology." IEEE Software, pp. 23-39, California, May, 1998.

[7] ZENDLER, Andreas. "A Preliminary Software Theory as Investigated by Published Experiments." Empirical Software Engineering, Vol. 6, no 2, pp. 161-180, Kluwer Academic Publishers, Netherlands, June, 2001. 